\title{
Pyloric Atresia: An Unusual Presentation
}

JCR Nadine Milad, Sami Sanjad, Lama Charafeddine

Department of Pediatrics and Adolescent Medicine, American University of

Beirut Medical Center, Beirut, Lebanon.

\section{Abstract:}

Pyloric atresia is a rare condition, comprising less than $1 \%$ of all intestinal atresias, with a reported incidence of $1 / 1,00,000$ newborns [1-3]. The usual presenting symptoms include non-bilious vomiting and abdominal distension [3-5]. Delay in diagnosis may occur since the non-bilious vomiting may be attributed mistakenly to gastroesophageal reflux. Complications resulting from such delays include aspiration pneumonia, recurrent lung infections, septicemia, severe metabolic derangements, and gastric perforation $[1,2,6]$. Here we present a 6-day-old female with pyloric atresia who presented with severe dehydration, hypotension, electrolyte abnormalities, and metabolic acidosis. After clinical stabilization and correction of biochemical abnormalities, the patient underwent surgical correction and had a smooth post-operative course. Currently she is 2 years and 3 months old, and has reached her expected developmental milestones.

Key words: Acidosis, Gastric Outlet Obstruction, Intestinal Atresia, Pneumonia, Pylorus.

\section{Introduction}

Pyloric atresia is a rare pathology, with an incidence of 1 in $1,00,000$ newborns. To date few case reports and small case series exist in the literature $[1,2,7]$. Due to the rarity of the lesion and because of our patient's severe and unusual clinical presentation, we chose to report it and discuss the specific laboratory abnormalities, steps leading to the diagnosis, management, and outcome.

\section{Case Report}

A 6-day-old female neonate presented to our emergency department due to hypoactivity and poor oral intake $(10 \mathrm{ml}$ of milk daily). She also had several episodes of yellowish, non-bilious vomiting and scant meconium. Urine output was not recorded. The baby was very lethargic, sleeping upto eight hours at a time, and often not waking up for feeds. She was seen by a physician on her third day of life and was diagnosed with gastro-esophageal reflux.

Prenatal history revealed a 26-year-old $G_{1} P_{0}$ mother, who was toxoplasma non-immune, negative hepatitis $B$ surface antigen and had a normal diabetes screen. Pregnancy was uncomplicated, however prenatal ultrasound revealed moderate polyhydramnios and a prominent fetal stomach. Family history was

\section{Corresponding Author: Dr. Lama Charafeddine}

Email: Ic12@aub.edu.lb

Received: November 20, 2015 | Accepted: February 12, 2016 | Published Online: March 10, 2016

This is an Open Access article distributed under the terms of the Creative Commons Attribution License (creativecommons.org/licenses/by/3.0)

Conflict of interest: None declared | Source of funding: Nil | DOl: http://dx.doi.org/10.17659/01.2016.0025 
remarkable for parents being first cousins. She was born at 38 weeks of gestation by normal vaginal delivery at a local hospital; her birth weight was 2400 grams and she did not cry for the first 3 minutes, but subsequently improved on flow oxygen. APGAR scores were 6 and 9 at 1 and 5 minutes respectively. She was discharged home on her second day of life.

\section{Examination revealed a lethargic,} hypoactive, and hypothermic newborn with a weak cry; blood pressure was $32 / 17 \mathrm{~mm} \mathrm{Hg}$, heart rate 156 beats per minute, respiratory rate $50 / \mathrm{min}$, and oxygen saturation $87 \%$ on room air. Weight was 1900 grams, which equals 20\% weight loss compared to her birth weight. She had Kussmaul breathing pattern, and showed signs of severe dehydration including sunken eyes and depressed fontanelles, greater than 3-second capillary refill, and tenting and dryness of the skin. Heart and lungs examination showed no abnormality. The abdomen was severely distended, with decreased to absent bowel sounds. She also had occasional staring episodes associated with desaturation.

Initial work-up included: blood gases, CBC, electrolytes, total serum protein, uric acid, and liver function tests. Metabolic work-up was also performed. Blood and urine cultures were sent before starting broad-spectrum antibiotics. Urine analysis and urine electrolytes were also assessed. Venous blood gases showed severe metabolic acidosis: $\mathrm{pH} 7.15, \mathrm{pCO}_{2} 26 \mathrm{mmHg}, \mathrm{pO}_{2} 45 \mathrm{mmHg}$, base deficit: 15, bicarbonate $9 \mathrm{mEq} / \mathrm{L}$, and $\mathrm{O}_{2}$ $72 \%$ [Table 1]. Initial blood chemistry was glucose $239 \mathrm{mg} / \mathrm{dL}$, BUN $109 \mathrm{mg} / \mathrm{dL}$, creatinine $7.1 \mathrm{mg} /$ $\mathrm{dL}$, sodium $147 \mathrm{mmol} / \mathrm{L}$, potassium greater than $8.5 \mathrm{mmol} / \mathrm{L}$, chloride $82 \mathrm{mmol} / \mathrm{L}$, carbon dioxide $8 \mathrm{mmol} / \mathrm{L}$, magnesium $3.8 \mathrm{mg} / \mathrm{dL}$, calcium $9.6 \mathrm{mg} /$ $\mathrm{dL}$, and phosphate $>20.7 \mathrm{mg} / \mathrm{dL}$, uric acid 34.4 $\mathrm{mg} / \mathrm{dL}$, anion gap $57 \mathrm{mmol} / \mathrm{L}$, serum osmolality $350 \mathrm{mOsm} / \mathrm{kg}$ and $\mathrm{BUN} /$ creatinine ratio was 17.4. Urine analysis revealed a $\mathrm{pH}$ of 7 , specific gravity of 1.010, protein +2 , and glucose of +2 . Urine osmolality was $334 \mathrm{mOsm} / \mathrm{kg}$. FENA and urine protein to creatinine ratio were calculated and were $44.6 \%$ and 2.2 respectively. $C B C$, liver function tests, albumin, and initial metabolic work up (ammonia, pyruvate, lactic acid, and serum ketone bodies) showed no significant abnormality. $C$ reactive protein was normal. Chest $X$-ray was normal. Abdominal film revealed extremely large lucency occupying most of the abdominal cavity likely representing a dilated stomach, with no air seen in the small or large bowel loops [Fig.1].

In summary, initial assessment revealed hypernatremia, hyperkalemia, hypochloremia, high anion gap metabolic acidosis, hypovolemia, and hyperglycemia with evidence of severe renal impairment. This constellation of findings is not characteristic of a specific pathology. Therefore, a broad range of differential diagnoses was considered including infectious, metabolic, renal, endocrinological, and gastrointestinal etiologies.

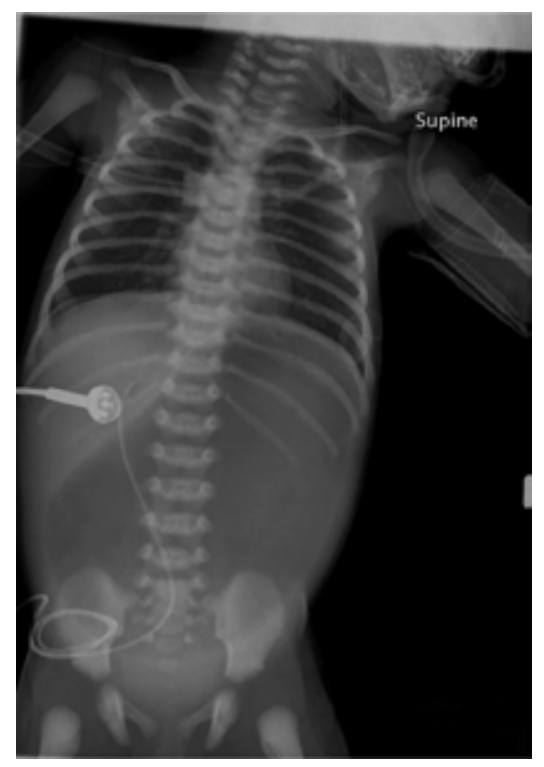

Fig.1: Abdominal film: large lucency occupying most of the abdominal cavity likely represents a dilated stomach, with no air seen in the small or large bowel loops. 
Sepsis was excluded after negative urine and blood cultures, and rapid improvement after hydration and correction of electrolyte abnormalities. Metabolic disease was ruled out by the normal arterial ammonia, lactate, pyruvate and normal urine organic acid analysis and neonatal screen. The acute kidney injury was secondary to severe dehydration and ensuing renal hypoperfusion. Intrinsic renal disease was excluded by a normal kidney ultrasound, and renal function normalized by sixth day of intravenous fluid therapy.

Neonatal diabetes was also considered briefly due to the hyperglycemia, acidosis, and dehydration. In the first two days of hospitalization insulin drip was required to control glucose level, after which hyperglycemia resolved without recurrence. Congenital adrenal hyperplasia could explain the shock-like presentation, hyperkalemia, and hypovolemia; but did not explain the hyperglycemia and lack of ambiguous genitalia. The history of non-bilious vomiting and polyhydramnios coupled with the abdominal distension on physical exam, hypochloremia, and findings on abdominal film ('single bubble sign'); were highly suspicious of gastric outlet obstruction. Despite the unusual finding of metabolic acidosis, a gastrointestinal etiology became the most likely differential.

In the emergency room central access was obtained, and intravenous fluid resuscitation $(20 \mathrm{ml} / \mathrm{kg}$ normal saline pushes) was administered. She was intubated to secure the airway due to the respiratory distress and severe metabolic acidosis. Nasogastric decompression was performed revealing bloodtinged milk, and she was transferred to the neonatal intensive care unit for further management. With progressive hydration serial arterial blood gases showed improvement in the metabolic acidosis [Table 1]. Similar improvements were seen in the electrolyte abnormalities, anion gap, and corrected bicarbonate [Fig.2]. Corrected bicarbonate is a calculation performed in the setting of anion gap metabolic acidosis to reveal an accompanying non-gap metabolic acidosis or metabolic alkalosis. If the corrected bicarbonate is above normal, then a concomitant primary metabolic alkalosis is present, as was the case seen with our patient. After stabilization, and on the fifth day of hospitalization, she underwent a gastrograffin swallow, which revealed complete gastric outlet obstruction [Fig.3]. A gastrograffin enema showed normal configuration of the rectum, sigmoid colon, colon, and small intestines [Fig.4]. On the sixth day of hospitalization, exploratory laparotomy was performed and she was found to have a completely obstructed pylorus, with complete partition of the pyloric canal and thickened mucosal and muscularis diaphragm consistent with type 2 pyloric atresia. Gastroduodenostomy was performed without complications.

Table 1: Serial Blood Gases

\begin{tabular}{|c|c|c|c|c|c|c|c|c|c|}
\hline Date & Time & $\begin{array}{l}\text { Ventilator Settings } \\
(\mathrm{PIP} / \mathrm{PEEP} \mathrm{RR} \mathrm{FiO})_{2} \text { * }\end{array}$ & Sample & $\mathrm{pH}$ & $\mathrm{pCO}_{2}$ & $\mathrm{pO}_{2}$ & Deficit & $\mathrm{HCO}_{3}$ & $\mathrm{O}_{2} \%$ \\
\hline Day 1 & $6 \mathrm{am}$ & Room Air & Venous & 7.15 & 26 & 45 & -15 & 9 & 72 \\
\hline Day 1 & $9 \mathrm{am}$ & $12 / 43530 \%$ & Arterial & 7.37 & 32 & 86 & -6 & 18 & 97 \\
\hline Day 1 & $2 \mathrm{pm}$ & $12 / 43030 \%$ & Arterial & 7.31 & 40 & 145 & -6 & 20 & 99 \\
\hline Day 2 & $6 \mathrm{am}$ & $11 / 43525 \%$ & Arterial & 7.43 & 30 & 96 & -3 & 19 & 98 \\
\hline
\end{tabular}

*PIP: Positive Inspiratory Presurre, PEEP: Positive End Expiratory Pressure, RR: Respiratory Rate, FiO ${ }_{2}$ : Fraction of inspired oxygen. 


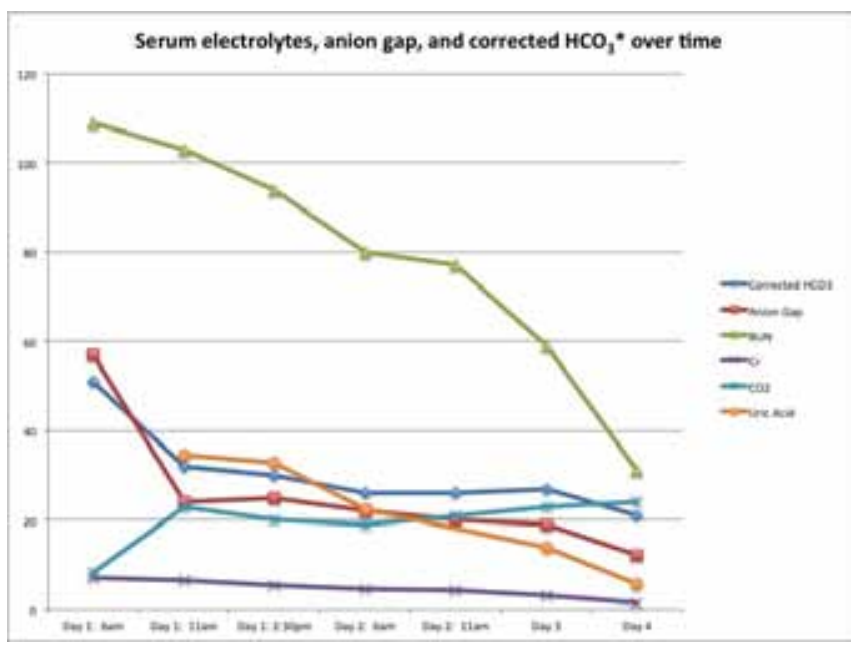

Fig.2: Serum electrolytes, anion gap, and corrected $\mathrm{HCO}_{3} *$ over time.

*Corrected $\mathrm{HCO}_{3}$ formula: Measured $\mathrm{HCO}_{3}$ + Anion Gap - 15 (Note: A value of -15 was used in the formula, since in neonates a normal anion gap can reach $15 \mathrm{mEq} / \mathrm{L}$ )

Corrected $\mathrm{HCO}_{3}(\mathrm{mEq} / \mathrm{L})$; anion Gap (mEq/L); BUN (Blood Urea Nitrogen) (mg/dL); $\mathrm{Cr}$ (Creatinine) $(\mathrm{mg} / \mathrm{dL}) ; \mathrm{CO}_{2}$ (carbon dioxide) (mEq/L); Uric Acid (mg/dL).

The post-operative course was relatively smooth. Feeding was initiated on the $4^{\text {th }}$ postoperative day, and full enteral feedings were reached within two weeks. By that time she was 28 days of age, and had reached a weight of 2350 grams. Currently she is a healthy 2 years 3 months old child who is thriving with normal developmental milestones.

\section{Discussion}

Our patient's late presentation resulted in the unusual finding of high anion gap metabolic acidosis. The etiologies of high anion gap metabolic acidosis are well documented in the literature, and include lactic acidosis, ketoacidosis, renal failure, toxin ingestion, and organic acidemia secondary to metabolic disorders. The expected metabolic alkalosis associated with pernicious vomiting due to her pyloric atresia was masked by the severe acute

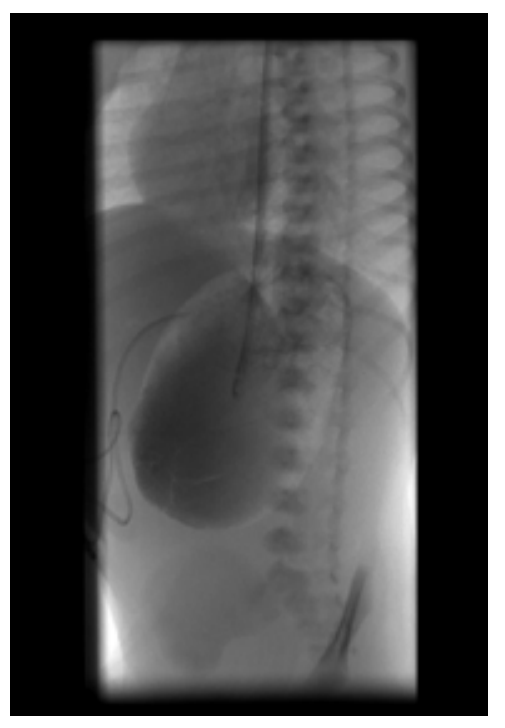

Fig.3: Gastrograffin swallow: complete gastric outlet obstruction.

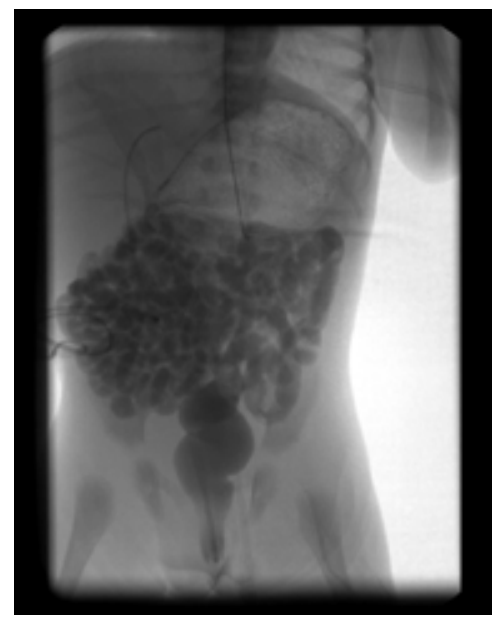

Fig.4: Gastrograffin enema: Normal configuration of the rectum, sigmoid colon, colon, and small intestines.

kidney injury resulting from extreme dehydration, hypovolemia and renal hypoperfusion. This acute uremia was the main cause of her severe anion gap metabolic acidosis. Lactate level was slightly elevated but is unlikely to have contributed to the gap acidosis. 
Congenital anomalies of the gastrointestinal tract a relatively common, however congenital pyloric atresia is a rare anomaly accounting for less that $1 \%$ of all intestinal atresias [8]. The incidence is equal among male and female neonates [1]. There are three anatomic variants of pyloric atresia; Type 1 the pyloric membrane or web is the most common accounting for $57 \%$ of the cases. In Type 2 (34\%) a fibrous solid cord replaces the pyloric canal, and in Type $3(9 \%)$ there is a complete disconnection between the stomach and duodenum [9]. Our case index had a type 2 lesion.

Patients usually present soon after birth due to non-bilious vomiting $[2,10]$, which is the most common clinical feature at presentation $1100 \%$ of cases) in addition to epigastrium distension $(68 \%$ of cases) [5]. In a case series by Llce et al., the condition was often associated with a low birth weight [1]. Prenatally the diagnosis is often suspected due to polyhydramnios (63\% of cases) [5]. Fetal ultrasound also often shows a dilated stomach and at times fluid distension of esophagus $[6,8,9]$. Postnatal diagnosis also relies on imaging, with abdominal radiograph showing the characteristic 'single-bubble' sign in up to $98 \%$ of the cases [5]. Postnatal ultrasound can also be performed and may show an elongated and echogenic pylorus with no patent channel $[2,6]$.

For all types of pyloric atresia the management is surgical. In Type 1, excision of the pyloric web is performed followed by a HeinekeMikulicz pyloroplasty; whereas Type 2 and 3 are treated by gastroduodenostomy [8]. Our patient's Type 2 lesion was corrected as recommended by gastroduodenostomy. Pyloric atresia can occur as an isolated lesion, or can be associated with other anomalies in approximately 33\%-55\% of cases $[1,6,10]$. The most commonly associated abnormality is epidermolysis bullosa $[7,10,11]$. Isolated congenital pyloric atresia has an excellent prognosis with appropriate surgical management, however the presence of associated anomalies negatively impact morbidity and mortality [6].

\section{References}

1. Ilce Z, Erdogan E, Kara C, Celayir S, Sarimurat $N$, Senyüz O, et al. Pyloric atresia: 15-year review from a single institution. J Pediatr Surg. 2003;38(11):1581-1584.

2. Okoye B, Parikh D, Buick R, Lander A. Pyloric atresia: Five new cases, a new association, and a review of the literature with guidelines. J of Pediatr Surg. 2000;35(8):1 242-1 245.

3. Muller $M$, Morger R, Engert J. Pyloric atresia: a report of four cases and review of literature. Pediatr Surg Int. 1990;5:276-279.

4. Andriessen MJ, Matthyssens LE, Heij HA. Pyloric atresia. J of Pediatr Surg. 2010;45(12):24702472.

5. Parshotam G, Ahmed S, Gollow I. Single or double bubble: Sign of trouble! Congenital pyloric atresia: Report of two cases and review of literature. J Paediatr Child Health. 2007;43(6):502-503.

6. Merrow AC, Frischer JS, Lucky AW. Pyloric atresia with epidermolysis bullosa: fetal MRI diagnosis with postnatal correlation. Pediatr Radiol. 2013;43(12):1656-1661.

7. Al-Salem A. Congenital pyloric atresia and associated anomalies. Pediatr Surg Int. 2007;23(6):559-563.

8. Al-Salem A, Abdulla $M$, Kothari $M$, Naga M. Congenital pyloric atresia, presentation, management, and outcome: A report of 20 cases. J Pediatr Surg. 2014;49(7):1078-1082.

9. Joshi P, Mundada D, Shetty S, Oak S, Parelkar $S$, Kapadnis $S$, et al. Pyloric atresia: Three cases and review of literature. Afr J Paediatr Surg. 2014;11(4):362-365.

10. Ksia A, Zitouni H, Zrig A, Laamiri R, Chioukh F, Ayari $E$, et al. Pyloric atresia: $A$ report of ten patients. Afr J Paediatr Surg. 2013;10(2):192-194.

11. Farmakis SG, Herman TE, Siegel MJ. Congenital pyloric atresia, type $B$; with junctional epidermolysis bullosa. J of Perinatol. 2014;34(7):572-573. 